\title{
Profitability of Technical Trading Rules on the Baltic Stock Markets*
}

\author{
Carl Lönnbark and Albina Soultanaeva \\ Department of Economics, Umeå University \\ SE-901 87 Umeå, Sweden
}

email: carl.lonnbark@econ.umu.se and albina.soultanaeva@econ.umu.se

\begin{abstract}
In this note we study whether simple technical trading rules are profitable on the three Baltic stock markets. To statistically assess our findings we consider the conventional $t$-test and a block-bootstrap procedure. The two evaluation methods give conflicting results. The $t$-test supports some of the rules, while the block-bootstrap does not.
\end{abstract}

Key Words: Baltic stock markets, technical trading rules, block bootstrap.

JEL Classification: G10, G14, G17.

Umeå Economic Studies 761, 2008

${ }^{*}$ The financial support from the Wallander-Hedelius Foundation is gratefully acknowledged. Kurt Brännäs is thanked for valuable comments and suggestions. 


\section{Introduction}

In their seminal paper, Brock et al. (1992) found support for technical trading rules on the Dow Jones Index. Following their study the interest in testing the profitability of technical trading rules has grown considerably. In particular, the emerging markets have attracted interest. Ratner and Leal (1999) found that technical trading rules do not have widespread ability to predict returns in ten large emerging markets in Asia and Latin America, while Parisi and Vasquez (2000) and Gunasekarage and Power (2001) found support for the profitability of technical trading rules in the Chilean stock market and four emerging South Asian Markets, respectively. Chang et al. (2004) also found that technical trading rules are profitable on some emerging equity markets. For more developed markets, Bokhari et al. (2005) found that the smaller the size of the company on FTSE 100, FTSE 250 and FTSE Small Cap the higher the predictive ability of technical trading rules. In this note, we are interested in studying whether technical trading rules are profitable on the Baltic stock markets. In particular, we evaluate ten different variable length moving average rules on index data from the three markets.

A technical rule generates excess profit over a buy-and-hold strategy if returns on buy days are significantly higher than returns on sell days. To assess whether this is the case for a particular market and rule several methods have been used in the literature. An obvious choice is to use the conventional $t$-test, but due to such properties of stock returns as fat tails, autocorrelation and conditional heteroskedasticity this approach may give erratic conclusions. An alternative approach, based on the bootstrap methodology of Efron (1979), was first employed by Brock et al. (1992). The most popular version of the method bootstrap the actual returns. However, as the validity of this bootstrap methodology crucially depends on the assumption of identically and independently distributed returns this approach may be questioned as well. In this paper, we use the block-bootstrap procedure of Künsch (1989), that takes into account the dependence structure in the data. To our knowledge this has not been done previously to evaluate the profitability of technical trading rules.

\section{Technical Rules and Methodology}

The variable length moving average rules (VMA) we consider involve comparison of short and long term moving averages of an index. A buy (sell) signal is emitted if the short term moving average crosses the long term from below (above). The rule may be modified by using a confidence band of $b$ percent. For moving average rules with a confidence band, buy signals are emitted when the short term moving average crosses the long term by $b$ percent and vice versa for sell signals. Here, we consider five different combinations for short and long term moving averages; (1 day, 50 days), $(1,150),(5,150),(1,200),(2,200)$. 
Each combination is evaluated with and without a confidence band of one percent, for a total of ten different VMA rules. For the no band case each day will be classified as either a buy or a sell day, while some days will be classified as neutral when using a confidence band.

If technical analysis has predictive power regarding stock price movements, i.e. if it generates profits in excess of a buy-and-hold strategy, returns on buy-days should be higher than returns on sell-days.

To assess whether returns on buy and sell days differ significantly we use $t$-tests ${ }^{1}$ and a block-bootstrap procedure. In the latter procedure the actual index return series of $T$ observations is divided into $B$ overlapping blocks of length $l$. The blocks, $B_{t}=$ $\left\{r_{t}, r_{t+1}, \ldots r_{t+l-1}\right\}$ for $t=1,2, \ldots,(T-l+1)$, are drawn randomly with replacement, generating an alternative realization of returns. The rules are then applied to the implied index series. For each trading rule we then compute sample means of the bootstrap returns conditional on buy and sell signals as well as unconditionally. We repeat the procedure 500 times. This is the number of replications used in previous studies and we conjecture that it is sufficient for generating a reliable empirical distribution of returns in our application as well.

The literature offers no clear guidance regarding the choice of the block length other than that it is important for the chosen blocks to contain the dependence structure in the data. In this paper, we use a block length of five trading days.

How bootstrap $p$-values are calculated and interpreted is best described by an example. To test whether returns on buy days are higher than on sell days, we calculate the fraction of replications with a higher mean return on "buy" days than on "sell" days. This fraction should exceed $1-\alpha$ for a significant result at level $\alpha$.

\section{Results}

The data used in this paper are capitalization weighted daily stock price indices of the Riga, Tallinn and Vilnius stock exchanges. ${ }^{2}$ The dataset covers January 3, 2000 to October, 5, 2007, for a total of $T=2025$ observations. The rules were applied to the daily index

\footnotetext{
${ }^{1}$ We use the $t$-statistics in Brock et al. (1992):

$$
t=\frac{\mu_{r}-\mu}{\left(\sigma^{2} / N+\sigma^{2} / N_{r}\right)^{1 / 2}}
$$
}

where $\mu_{r}$ and $N_{r}$ are the mean return and the number of signals for the buys and sells, and $\mu$ and $N$ are the unconditional mean and number of observations. $\sigma^{2}$ is the unconditional variance. For the spread, the $t$-statistic is

$$
\frac{\mu_{b}-\mu_{s}}{\left(\sigma^{2} / N_{b}+\sigma^{2} / N_{s}\right)^{1 / 2}}
$$

\footnotetext{
${ }^{2}$ Indices were collected from the stock exchanges official web site www.omxgroup.com.
} 
log-returns. In Table 1 we give results for each index and rule.

We first note that for the Riga index (Panel A) all $t$-statistics are small and insignificant at conventional levels indicating that the rules does not appear to generate returns in excess of the buy and hold strategy. The block-bootstrap results support this finding as the $p$-values are in the range 0.34 to 0.67 .

For Tallinn and the $(1,50,0)$ and $(1,50,0.01)$ rules the $t$-test indicate higher mean returns. The mean daily buy return is 0.131 , on average, and the mean sell return is 0.019 , on average. Interestingly however, this finding is not supported by the block-bootstrap results.

For Vilnius the mean daily buy return is on average 0.16 and the mean sell return is -0.020 on average and significantly lower than the unconditional mean. The average spread is 0.177 and significantly different from zero on a 5 percent level, when looking at the $t$-test. The profitability of rules decreases with the number of days used for the short and long term moving average; the $(1,50,0)$-rule is the most profitable. The $p$-values for Vilnius, presented in the final columns of the Table 2, Panel C, are similar to ones for Riga and Tallinn. That is, the block-bootstrap $p$-values for Vilnius, in contrast with the $t$-test results do not support the hypothesis of profitability of technical trading rules.

Summarizing, in this note we demonstrate that the choice of a methodolody for testing the profitability of technical trading rules is crucial for the results. Even when not taking into account the transaction costs for trading, the rules exhibit no profitability when the testing method accounts for dependence structure in the data.

\section{References}

Bokhari, J., Cai, C., Hudson, R., Keasey, K., 2005. The predictive ability and profitability of technical trading rules: does company size matter? Economics Letters 86, 21-27.

Brock, W., Lakonishok, J., LeBaron, B., 1992. Simple technical trading rules and the stochastic properties of stock returns. Journal of Finance 47, 1731-64.

Chang, E. J., Lima, E. J. A., Tabak, B. M., 2004. Testing for predictability in emerging equity markets. Emerging Markets Review 5, 295-316.

Efron, B., 1979. Bootstrap methods: Another look at the jackknife. The Annals of Statistics $7,1-26$.

Gunasekarage, A., Power, D. M., 2001. The profitability of moving average trading rules in South Asian stock markets. Emerging Markets Review 2, 17-33.

Künsch, H. R., 1989. The jackknife and the bootstrap for general stationary observations. The Annals of Statistics 17, 1217-1241. 
Parisi, F., Vasquez, A., 2000. Simple technical trading rules of stock returns: evidence from 1987 to 1998 in Chile. Emerging Markets Review 1, 152-164.

Ratner, M., Leal, R. P. C., 1999. Tests of technical trading strategies in the emerging equity markets of Latin America and Asia. Journal of Banking \& Finance 23, 1887-1905. 
Table 1: Results. The first column indicate rule as (short, long, band). Mean returns on buy and sell days are reported in the second and third columns, whereas the mean spreads are given in the fourth column. The corresponding $t$-statistics are given in parantheses. The columns five to seven show the corresponding block-bootstrap $p$-values.

\begin{tabular}{|c|c|c|c|c|c|c|}
\hline Rule & $\overline{\mu_{\text {buy }}^{t}}$ & $\mu_{\text {sell }}^{t}$ & $\mu_{\text {spread }}^{t}$ & $\overline{\mu_{\text {buy }}^{b}}$ & $\mu_{\text {sell }}^{b}$ & $\overline{\mu_{\text {spread }}^{b}}$ \\
\hline \multicolumn{7}{|c|}{ Panel A: Riga } \\
\hline$\overline{(1,50,0)}$ & $0.105(0.132)$ & $0.070(-0.404)$ & $0.035(0.478)$ & 0.644 & 0.340 & 0.660 \\
\hline$(1,50,0.01)$ & $0.117(0.334)$ & $0.093(-0.062)$ & $0.024(0.284)$ & 0.634 & 0.338 & 0.670 \\
\hline$(1,150,0)$ & $0.098(0.000)$ & $0.105(0.081)$ & $-0.007(-0.078)$ & 0.636 & 0.374 & 0.630 \\
\hline$(1,150,0.01)$ & $0.095(-0.062)$ & $0.128(0.339)$ & $-0.033(-0.363)$ & 0.632 & 0.372 & 0.638 \\
\hline$(5,150,0)$ & $0.099(0.020)$ & $0.101(0.037)$ & $-0.002(-0.023)$ & 0.468 & 0.532 & 0.470 \\
\hline$(5,150,0.01)$ & $0.090(-0.150)$ & $0.084(-0.165)$ & $0.006(0.070)$ & 0.486 & 0.540 & 0.446 \\
\hline$(1,200,0)$ & $0.090(-0.163)$ & $0.090(-0.089)$ & $-0.001(-0.006)$ & 0.614 & 0.400 & 0.600 \\
\hline$(1,200,0.01)$ & $0.086(-0.236)$ & $0.142(0.456)$ & $-0.056(-0.571)$ & 0.624 & 0.394 & 0.608 \\
\hline$(2,200,0)$ & $0.093(-0.107)$ & $0.078(-0.230)$ & $0.015(0.163)$ & 0.568 & 0.440 & 0.564 \\
\hline$(2,200,0.01)$ & $0.096(-0.046)$ & $0.122(0.244)$ & $-0.026(-0.262)$ & 0.566 & 0.460 & 0.572 \\
\hline \multicolumn{7}{|c|}{ Panel B: Tallinn } \\
\hline$(1,50,0)$ & $0.156(1.805)$ & $-0.045(-3.059)$ & $0.201(4.225)$ & 0.822 & 0.162 & 0.832 \\
\hline$(1,50,0.01)$ & $0.164(1.960)$ & $-0.010(-2.061)$ & $0.175(3.299)$ & 0.824 & 0.186 & 0.826 \\
\hline$(1,150,0)$ & $0.128(1.061)$ & $0.010(-1.550)$ & $0.118(2.178)$ & 0.628 & 0.380 & 0.620 \\
\hline$(1,150,0.01)$ & $0.133(1.176)$ & $0.026(-1.121)$ & $0.106(1.782)$ & 0.626 & 0.408 & 0.602 \\
\hline$(5,150,0)$ & $0.118(0.763)$ & $0.043(-0.928)$ & $0.075(1.389)$ & 0.466 & 0.540 & 0.432 \\
\hline$(5,150,0.01)$ & $0.126(0.989)$ & $0.049(-0.732)$ & $0.077(1.298)$ & 0.452 & 0.546 & 0.452 \\
\hline$(1,200,0)$ & $0.123(0.918)$ & $0.019(-1.227)$ & $0.104(1.725)$ & 0.552 & 0.418 & 0.584 \\
\hline$(1,200,0.01)$ & $0.123(0.928)$ & $0.028(-1.008)$ & $0.095(1.490)$ & 0.574 & 0.478 & 0.530 \\
\hline$(2,200,0)$ & $0.118(0.776)$ & $0.040(-0.875)$ & $0.078(1.300)$ & 0.500 & 0.504 & 0.500 \\
\hline$(2,200,0.01)$ & $0.119(0.813)$ & $0.034(-0.915)$ & $0.085(1.338)$ & 0.494 & 0.526 & 0.482 \\
\hline \multicolumn{7}{|c|}{ Panel C: Vilnius } \\
\hline$(1,50,0)$ & $0.181(2.583)$ & $-0.062(-3.527)$ & $0.243(5.269)$ & 0.764 & 0.234 & 0.778 \\
\hline$(1,50,0.01)$ & $0.196(2.901)$ & $-0.039(-2.769)$ & $0.234(4.697)$ & 0.790 & 0.262 & 0.766 \\
\hline$(1,150,0)$ & $0.150(1.767)$ & $-0.016(-2.203)$ & $0.166(3.331)$ & 0.554 & 0.450 & 0.546 \\
\hline$(1,150,0.01)$ & $0.144(1.570)$ & $-0.019(-2.158)$ & $0.163(3.106)$ & 0.570 & 0.440 & 0.560 \\
\hline$(5,150,0)$ & $0.140(1.495)$ & $0.007(-1.722)$ & $0.134(2.687)$ & 0.452 & 0.580 & 0.414 \\
\hline$(5,150,0.01)$ & $0.149(1.704)$ & $-0.003(-1.838)$ & $0.152(2.897)$ & 0.492 & 0.570 & 0.438 \\
\hline$(1,200,0)$ & $0.150(1.775)$ & $-0.028(-2.313)$ & $0.178(3.392)$ & 0.538 & 0.470 & 0.532 \\
\hline$(1,200,0.01)$ & $0.153(1.836)$ & $-0.016(-2.002)$ & $0.169(3.104)$ & 0.544 & 0.508 & 0.504 \\
\hline$(2,200,0)$ & $0.149(1.738)$ & $-0.024(-2.234)$ & $0.172(3.294)$ & 0.514 & 0.496 & 0.488 \\
\hline$(2,200,0.01)$ & $0.150(1.761)$ & $-0.003(-1.758)$ & $0.153(2.822)$ & 0.512 & 0.530 & 0.454 \\
\hline
\end{tabular}

